\title{
A 0.35 THz Extended Interaction Oscillator based on Overmoded and Bi-Periodic Structure
}

\begin{tabular}{|c|c|}
\hline Journal: & Transactions on Electron Devices \\
\hline Manuscript ID & TED-2021-04-0933-R \\
\hline Manuscript Type: & Regular \\
\hline $\begin{array}{r}\text { Date Submitted by the } \\
\text { Author: }\end{array}$ & 18-Apr-2021 \\
\hline Complete List of Authors: & $\begin{array}{l}\text { Xu, Che; University of Electronic Science and Technology of China, } \\
\text { School of Physical Electronics } \\
\text { Meng, Lin; University of Electronic Science and Technology of China, } \\
\text { School of Physical Electronics } \\
\text { Paoloni, Claudio; Lancaster University, Engineering Department } \\
\text { Qin, Yu; University of Electronic Science and Technology of China, School } \\
\text { of Electronic Science and Engineering } \\
\text { Bi, Liangjie; University of Electronic Science and Technology of China, } \\
\text { School of Electronic Science and Engineering } \\
\text { Wang, Bin; University of Electronic Science and Technology of China, } \\
\text { School of Physical Electronics } \\
\text { Li, Hailong; University of Electronic Science and Technology of China, } \\
\text { Yin, Yong; University of Electronic Science and Technology of China, } \\
\text { School of Physical Electronics }\end{array}$ \\
\hline Area of Expertise: & $\begin{array}{l}\text { Klystrons, Microwave oscillators, Millimeter wave tubes, Terahertz } \\
\text { radiation }\end{array}$ \\
\hline
\end{tabular}

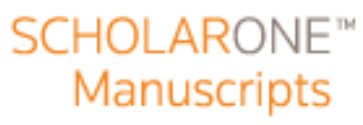




\title{
A 0.35 THz Extended Interaction Oscillator based on Overmoded and Bi-Periodic Structure
}

\author{
Che Xu, Lin Meng, Claudio Paoloni, Yu Qin, Liangjie Bi, Bin Wang, Hailong Li, and Yong Yin
}

\begin{abstract}
An improved topology of Extended interaction oscillators (EIO) is presented with enhanced efficiency at 0.35 THz. A bi-periodic ladder-type structure is used for optimizing the performance of the $\mathrm{TM}_{31}-2 \pi$ mode operation. The proposed topology is based on bi-periodic interaction gaps composed by staggered short and long slots. The bi-periodic interaction mechanism permits to operate the circuit with a standing-wave field between the $\pi$ and $2 \pi$ modes, thus providing the potential to combine the advantages of both $\pi$ and $2 \pi$ modes in terms both of coupling and output performance. The resulting transverse $\mathbf{T M}_{31}$ operating mechanism exhibits a good agreement with bi-periodic structure in terms of coupling performance, with the short slot length positively correlated with the value of effective characteristic impedance $M^{2} R / Q$. The optimization of $M^{2} R / Q$ and $Q_{e}$, the circuit demonstrates attractive coupling and output characteristics. A state-of-the-art value $M^{2} R / Q$ of $59.69 \Omega$ and a moderate $Q_{e}$ of $\mathbf{2 5 2 3 . 5 2}$ are achieved for the proposed ladder-type EIO. A RF power of $540 \mathrm{~W}$ is obtained with a $42.5 \mathrm{kV}, 0.6 \mathrm{~A}$ sheet beam, with $2.1 \%$ electronic efficiency at $0.35 \mathrm{THz}$.
\end{abstract}

Index Terms-Extended interaction oscillator (EIO), terahertz sources, vacuum electronics, bi-periodic structure.

\section{Introduction}

$\mathrm{T}$ ERAHERTZ vacuum electronic devices (VEDs) are highly potent radiation sources known, which promise to enable higher power and efficiency than laser or semiconductor sources [1-5]. Among such devices, compact and powerful extended interaction devices (EIDs) have shown great potential in plasma diagnostics, spectroscopy, and military applications due to their high gain per unit length [1, 6-12]. With the characteristics of both slow-wave structure (SWS) and coupled cavities, extended interaction oscillator and extended

This work was supported by the National Natural Science Foundatio $\mathrm{n}$ of China (No. 61671116, 61771096, 11905026), National Key Resea rch and Development Program of China (No.2019YFA0210202) and F undamental Research Funds for the Central Universities (No. ZYGX20 19Z006, ZYGX2019J012). The China Scholarship Council, P.R. China, is gratefully acknowledged for the support of $\mathrm{C}$. Xu. (Corresponding author: Lin Meng)

C. $\mathrm{Xu}$ is with the Vacuum Electronics National Laboratory, School of Electronic Science and Engineering, University of Electronic Science and Technology of China, Chengdu 610054, China, and also with the Engineering Department, Lancaster University, Lancaster LA1 4YW, U. K. (email: chexu1992@163.com)

L. Meng, Y. Yin, Y. Qin, L. Bi, B. Wang, and H. Li are with the Vacuum Electronic National Laboratory, School of Electronic Science and Engineering, University of Electronic Science and Technology of China, Chengdu 610054, China. (email: meng@uestc.edu.cn)

C. Paoloni is with the Engineering Department, Lancaster University, Lancaster LA1 4YW, U. K. (email: c.paoloni@lancaster.ac.uk) interaction klystron (EIO \& EIK) combine the advantages of traveling wave tubes (TWTs) and conventional multi-cavity klystrons.

Modern EID technology is demonstrated from 17 to 280 $\mathrm{GHz}$, with the capability to approaching $1 \mathrm{THz}$ [1]. On the basis of the conventional-transverse $2 \pi$ operation mode, EIDs have also evolved into higher-order modes in longitudinal ( $\pi$ mode) and transversal $\left(\mathrm{TM}_{\mathrm{n} 1}\right.$ modes) directions. Generally, $2 \pi$ mode EID is able to delivering high values of characteristic impedance $R / Q$, which compensate the ohmic loss at the increase of frequency. This is of importance for improving the electronic efficiency in $\mathrm{THz}$ band. For example, a $0.34 \mathrm{THz}$, $\mathrm{TM}_{31}-2 \pi$ mode EIK shows an electronic efficiency of 1.3\% [13]; a $0.67 \mathrm{THz}$ multi-cavity EIK has an efficiency goal of $0.5 \%$ [2]; and a $0.35 \mathrm{THz}$ high-order-mode EIO has a predicted electronic efficiency of $\sim 1 \%$ [3]. On the other hand, better coupling capabilities and mode isolation for the $\pi$-mode are major advantages. As a reference, a G-band bi-periodic EIK with a high value $M^{2} R / Q$ of $29.34 \Omega$ [14] demonstrated an efficiency target of $7.9 \%$ for stable operation in $\pi$-mode. The term ' $M$ ' refers to coupling coefficient. The effective characteristic impedance $M^{2} R / Q$ is the key parameter of EID technology [10]. To note that the more established $2 \pi$-mode operation has stable output characteristics but suffers from a lower coupling coefficient, while the $\pi$-mode usually exhibits high values of $Q_{e}$, the ratio of cavity energy storage to external circuit losses, and is therefore difficult to output because of the reduced weighting for the external circuit losses. The term $Q_{e}$ is the external quality factor.

Based on the above-mentioned electronic performances of $\pi$ and $2 \pi$ mode devices, the paper proposes a bi-periodic RF circuit with overmoded structure utilizing both $\pi$ and $2 \pi$ mode operation mechanisms. The proposed structure will show the following advantages.

Firstly, the transversal higher-order-mode scheme with $\mathrm{TM}_{31}$ distribution is adopted. In THz region, non-Brillouin thermal beam has a scalloped amplitude on the order of sub-millimeter wavelengths and the expansion of the longitudinal electron velocity prevents effective modulation [1]. Thus, an over-dimensioned high-order-mode structure is employed in order to overcome the size limitation of micro-circuits and to meet the requirements of high filling factor (beam current). Secondly, a strong coupled $2 \pi$ mode is established with a field distribution intermediate between conventional $\pi$ and $2 \pi$ modes. This will allow a trade-off between the stable output performance for the $2 \pi$ mode and the high coupling characteristics for the $\pi$ mode. Thirdly, the combination of an overmoded structure and a bi-periodic structure demonstrates 
TABLE I

BASIC PARAMETERS OF SEVERAL TYPICAL REPORTED EIDS

\begin{tabular}{ccccc}
\hline \hline Reference & Device & $\begin{array}{c}\text { Operation } \\
\text { mode }\end{array}$ & $\begin{array}{c}\text { Frequency } \\
(\mathrm{GHz})\end{array}$ & $\begin{array}{c}\mathrm{M}^{2} \mathrm{R} / \mathrm{Q} \\
(\Omega)\end{array}$ \\
\hline$[14]$ & EIK & $\pi$ & 219 & 29.34 \\
{$[15]$} & EIO & $2 \pi$ & 34 & 21.01 \\
{$[16]$} & EIK & $\pi$ & 220 & $\sim 15$ \\
{$[17]$} & EIK & $\pi$ & 35 & $\sim 6$ \\
{$[18]$} & EIK & $2 \pi$ & 94 & $<51$ \\
{$[19]$} & EIO & $2 \pi$ & 94 & $\sim 22$ \\
This paper & EIO & $2 \pi$-like & 352 & 59.69 \\
\hline \hline
\end{tabular}

strong coupling ability with a relatively higher $M^{2} R / Q$ value (59.69 $\Omega$ ) compared to other reported ladder-type EIDs [14-19]. For reference, the $M^{2} R / Q$ values of several EIDs are listed in Table I.

This paper will discuss on the electronic characteristics of the proposed circuit and its potential to powerful THz EIDs through numerical simulations.

\section{CIRCUIT DESCRIPTION AND STABILITY ANALYSIS}

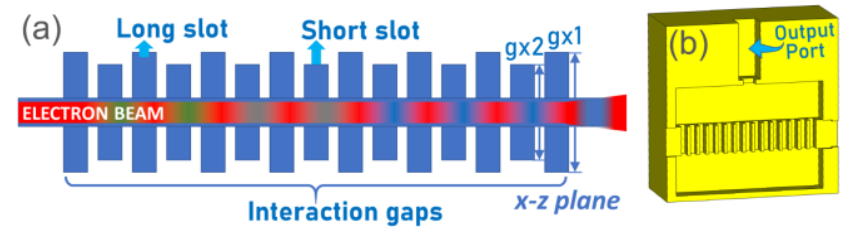

Fig. 1. (a) The cross-sectional schematic diagram of the EIO structure in $x-z$ plane and (b) a schematic of its 3D model.

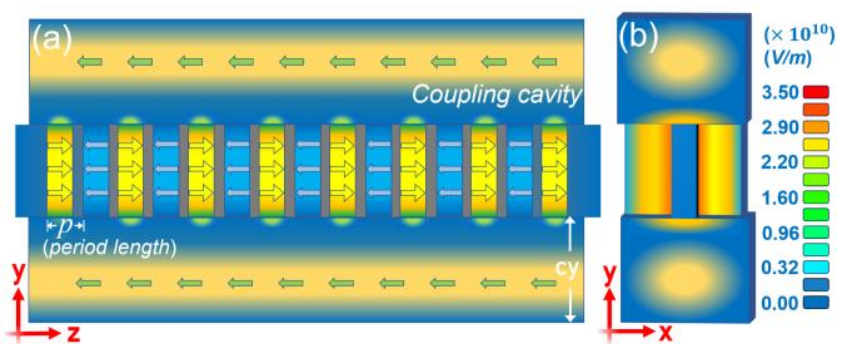

Fig. 2. The cross-sectional schematic diagrams of the EIO structure in (a) $y-z$ plane, and (b) $x-y$ plane. Their z-directional electric field components, $E_{z}$, are represented as color mapping.

Fig. 1 and Fig. 2 show the two-dimensional schematic of the proposed resonant circuit with an output structure that forms an EIO at $0.35 \mathrm{THz}$. It mainly consists of a SWS with seven short slots $(0.4 \times 0.5 \times 0.1 \mathrm{~mm})$, eight long slots $(0.5 \times 0.5 \times 0.1 \mathrm{~mm})$, two symmetrically distributed coupling cavities $(0.56 \times 0.69 \times 2.4 \mathrm{~mm})$ and a sheet beam tunnel (with cross-section area of $0.12 \times 0.5 \mathrm{~mm}$ ). The staggered long slots and short slots form the coupling gaps, which are the topology structure for constructing strong coupled $2 \pi$ mode circuits. Fig. 2(a) and Fig. 2(b) show the electric field distribution profiles with a cavity energy storage of $1 \mathrm{~J}$. In transversal direction ( $y$-direction), the three separate parts of $E_{z}$ field distributions are typical for the $\mathrm{TM}_{31}$ mode in an EIO [13, 20], which provides an over-dimensional structure for beam injection. In longitudinal direction ( $z$-direction), the electric field fills mainly the long slots, forming a $2 \pi$ mode distribution.

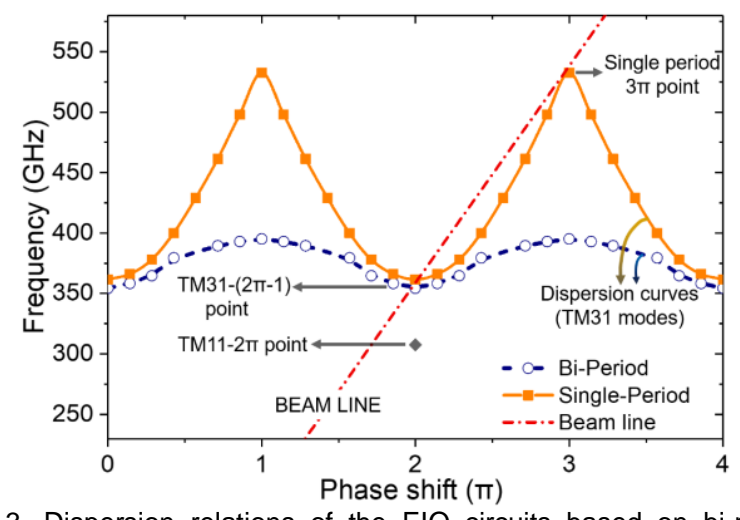

Fig. 3. Dispersion relations of the EIO circuits based on bi-period structure and single-period structure.

Typically, for a $2 \pi$ mode operation, there is a $2 \pi$ phase difference between the adjacent long slots which are also isolated from each other. In this case, the interaction gaps can be considered as rectangular waveguides that transmit $\mathrm{TE}_{10}$ waves [19]. Therefore, the cutoff frequency for such $\mathrm{TE}_{10}$ wave is determined by the long-side widths, for instance, $g x 1$ and $g x 2$ (in Fig. 1). If the long-side width decreases, the cutoff frequency of this rectangular waveguide increases and will exceeds $0.35 \mathrm{THz}$ when $g x$ is less than $0.43 \mathrm{~mm}$. At this point, we place short slots (less than $0.43 \mathrm{~mm}$ in width) between the long slots, and the $\pi$-phase electric field between adjacent $2 \pi$-phase fields will be expected to show weak transmission characteristics. The simulated cavity $E_{z}$ distribution is consistent with the above analysis, as shown in Fig. 2(a), the introduction of such a bi-period topology allows for the existence and propagation of a weak $\pi$-phase electric field in the short slots. In addition, the introduction of the short slot mechanism only increases the number of gaps and does not increase the machining accuracy requirements.

Each part of the circuit is designed to meet machining requirements of the UV-LIGA technique and electric discharge machining (EDM) [21, 22]. The overall length of the SWS is maintained below $3 \mathrm{~mm}$ and common sets of magnets are used with magnetic fields of approximately $1 \mathrm{~T}$.

Fig. 3 shows the dispersion diagram of the proposed circuit with/without short slot structure. The period length $p$ is set at $0.15 \mathrm{~mm}$ synchronised with a beam voltage of $\sim 42 \mathrm{kV}$. From the diagram, the $\mathrm{TM}_{31}$ mode of the bi-period circuit provides higher-frequency $354.25 \mathrm{GHz}$ compared to the $\mathrm{TM}_{11}$ mode at $307.65 \mathrm{GHz}$. Furthermore, with the specific bi-period design as described above, the dispersion characteristics of the circuit show a significant difference from the conventional single-period structure. Fig. 3 shows that the beamline relative to the synchronous condition crosses both $2 \pi$ and $3 \pi$ points in the case of single period, thus predisposing to parasitic mode competition. In contrast, the bi-period mechanism utilizes $\pi$-mode dispersion characteristics to keep the parasitic modes (such as $3 \pi$ mode) away from beamline, and more difficult to be excited. This selective mode isolation ensures stable operation at low noise levels.

The designed bi-periodic cavity possesses a number of discrete frequencies provided by the number of half-wavelengths between the end walls of the cavity, where $\mathrm{TM}_{31}-(2 \pi-1)$ and $\mathrm{TM}_{11}-2 \pi$ points are adjacent to the beam line 
TABLE II

BASIC PARAMETERS OF THE EIGEN-MODES FOR BI-PERIODIC STRUCTURE

\begin{tabular}{ccccc}
\hline \hline Mode & $\begin{array}{c}\text { Frequency } \\
(\mathrm{GHz})\end{array}$ & $\mathrm{R} / \mathrm{Q}(\boldsymbol{\Omega})$ & $\mathrm{Q}_{0}$ & $\begin{array}{c}\mathrm{M}^{2} \mathrm{R} / \mathrm{Q} \\
(\boldsymbol{\Omega})\end{array}$ \\
\hline $\mathrm{TM}_{31}-2 \pi$ & 354.25 & 139.39 & 980.29 & 59.69 \\
$\mathrm{TM}_{31}-2 \pi-1$ & 358.22 & 111.36 & 999.20 & 44.38 \\
$\mathrm{TM}_{11}-2 \pi$ & 307.65 & 184.57 & 949.35 & 21.01 \\
\hline \hline
\end{tabular}

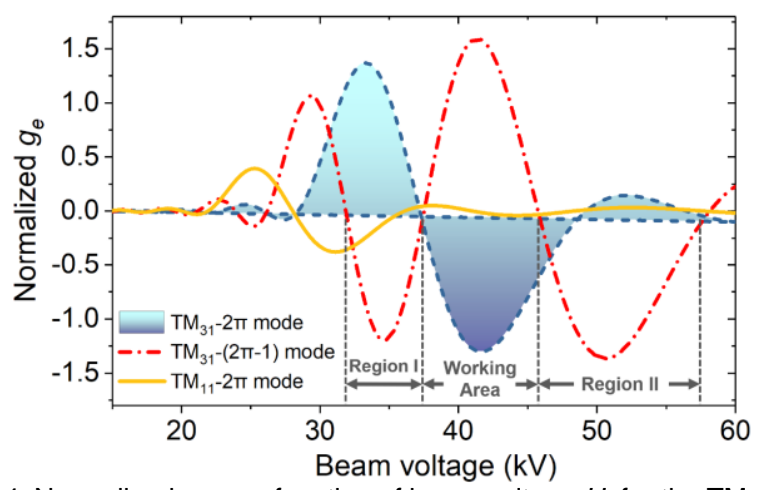

Fig. 4. Normalized $g_{e}$ as a function of beam voltage $U_{0}$ for the $T M_{31}-2 \pi$, $\mathrm{TM}_{31}-(2 \pi-1)$, and $\mathrm{TM}_{11}-2 \pi$ modes in the bi-periodic structure.

in Fig. 3. A distinguishing characteristic for an oscillation mode is the existence of an absolute resonance threshold, for instance a voltage range, above or below which the cavity will not oscillate. Once the resonance condition is satisfied, undesired mode-competitions caused by $\mathrm{TM}_{31}-(2 \pi-1)$ and $\mathrm{TM}_{11}-2 \pi$ modes may occur. When considering the beam loading in the circuit, the normalized beam-loading conductance $g_{e}$ is a key factor in the discussion of oscillation or amplification. A negative value of $g_{e}$ can satisfy the oscillation state. In this case, the energy exchange between the electron beam and circuit is such that the net energy flows to the circuit, which would form an oscillating positive feedback loop unless it is sufficiently loaded or sufficiently lossy. The normalized beam-loading conductance $g_{e}$ can be obtained from $[22,23]$

$$
g_{e}=\frac{1}{8} \frac{\beta_{e}}{\beta_{q}}\left[\left|M_{-}^{2}\left(\beta_{e}-\beta_{q}\right)\right|-\left|M_{+}^{2}\left(\beta_{e}+\beta_{q}\right)\right|\right],
$$

where $M$ can be expressed by [23,24]

$$
M\left(\beta_{e} \pm \beta_{q}\right)=\frac{\int_{-\infty}^{\infty} E(z) e^{j\left(\beta_{e} \pm \beta_{q}\right) z} d z}{\int_{-\infty}^{\infty}|E(z)| d z} .
$$

$\beta_{e}$ and $\beta_{q}$ are the propagation constants for DC beam and reduced plasma, respectively. The variation of $g_{e}$ with voltage of the $\mathrm{TM}_{31}-2 \pi, \mathrm{TM}_{31}-(2 \pi-1)$, and $\mathrm{TM}_{11}-2 \pi$ modes are shown in Fig. 4, with their characteristic parameters shown in Table II. When the beam voltage is in the range of 37.5 to $45.7 \mathrm{kV}$, the oscillation conditions for the $\mathrm{TM}_{31}-(2 \pi-1)$ and $\mathrm{TM}_{11}-2 \pi$ modes will not be satisfied ( $g_{e}$ is positive), as shown in Fig. 4. This will ensure stable single-mode operation of the $\mathrm{TM}_{31}-2 \pi$ mode synchronized with $\sim 42 \mathrm{kV}$. However, as shown in Fig.4, once the voltage has shifted outside the above-mentioned voltage range, the undesired $\mathrm{TM}_{31}-(2 \pi-1)$ mode is also potentially excited, and thus, operations in Region I and II should be avoided.

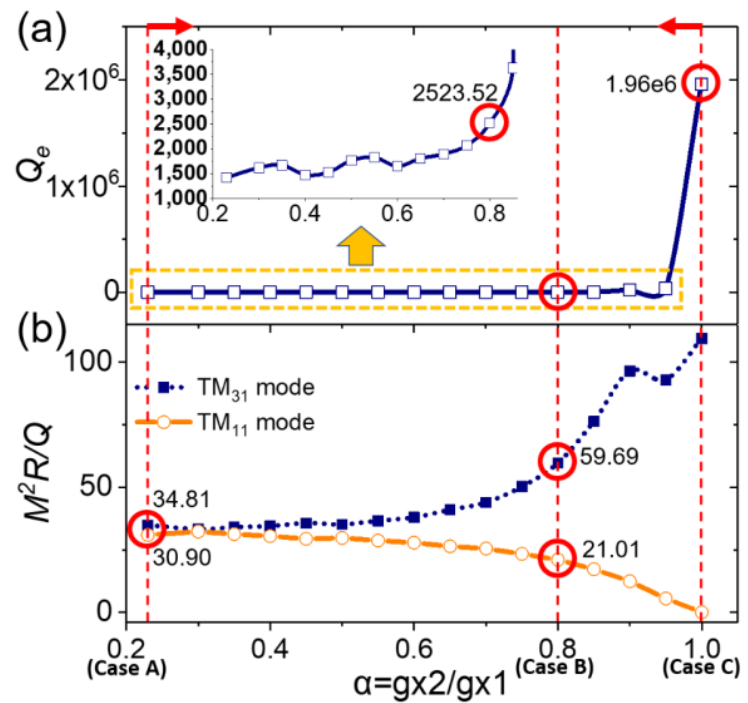

Fig. 5. Variations of (a) $Q_{e}$ and (b) $M^{2} R / Q$ for the proposed $R F$ circuit as a function of length factor $\alpha$. In this case, the length of long slot, gx1, remains the same.

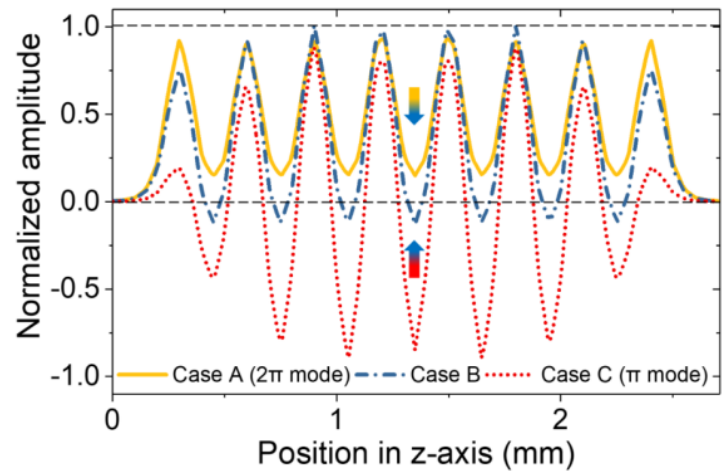

Fig. 6. Axial electric field components of the beam axis at $\alpha$ less than 0.24 (Case A), equal to 0.8 (Case B), and equal to 1.0 (Case C), respectively.

\section{SIMULATIONS AND ANALYSIS}

Extensive 3-D simulations and calculations for the proposed structure have been performed by CST-Microwave Studio [25] to demonstrate its behaviour and establish the strong coupled $2 \pi$ mode. Copper is used as lossy metal material. The characteristic parameters $Q_{e}$ and $M^{2} R / \mathrm{Q}$ as a function of length factor $\alpha=g x 2 / g x l$ are shown in Fig. 5(a) and 5(b), $Q_{e}$ is the ratio of cavity energy storage to external circuit losses. $R / Q[11$, $23,24]$ can be expressed by

$$
R / Q=\frac{\left(\int_{-\infty}^{\infty}\left|E_{z}\right| d z\right)^{2}}{2 w W_{s}},
$$

where angular frequency $w$ and total energy storage $W_{s}$ are the measure of $E_{z}$ field.

Three structures based on different length factor $\alpha$ were analyzed (results in Fig. 5). Case A (single-period, $\alpha$ less than 0.24 ), B (staggered bi-period, $\alpha=0.8$ ), and $\mathrm{C}$ (uniformed bi-period, $\alpha=1$ ), respectively, with their corresponding axial field components are shown in Fig. 6. Accordingly, their field distributions shift gradually with $\alpha$ from $2 \pi$ to $\pi$ modes. From the definition of $Q_{e}$, a relatively small value of $Q_{e}$ risks reducing the energy storage in the cavity (represented by Case A in Fig. 5), while a too large $Q_{e}$ is highly detrimental to the RF output 
(represented by Case C). Nevertheless, this still suggests that the $2 \pi$ mode (Case A) with a lower $Q_{e}$ value is more favorable for the output to the external circuit. From the other perspective, $M^{2} R / Q$, which is the key parameter of EID technology [10], appears to have a positive correlation with $\alpha$ (Fig. 5(b)). Such correlation not only implies that the structure A has an $M^{2} R / Q$ value greater than $34.81 \Omega$, but also that at the increases of $\alpha$ (when the field profile approaching $\pi$ mode) a $M^{2} R / Q$ value could reach $109 \Omega$, as shown in Fig. 5(b). Not only that, but the used $\mathrm{TM}_{31}$ mechanism appears to be more compatible with the bi-periodic topology, in contrast to the $\mathrm{TM}_{11}$ mechanism which provides an $M^{2} R / Q$ value below $30.90 \Omega$ in Fig. 5(b). Where the value of $M^{2} R / Q$ is merely $21.01 \Omega$ when $\alpha=0.8$. Similarly, for $\mathrm{TM}_{11}$ coupling pattern, two recently reported bi-periodic EIKs for $\pi$-mode operation achieved $M^{2} R / Q$ values of $\sim 29.34 \Omega$ [14] and $\sim 15.07 \Omega[16]$, respectively.

From the above discussion, trade-offs should be made in obtaining favorable values of $M^{2} R / Q$ and $Q_{e}$. In the case C corresponding to $\pi$-mode, the near-infinite $Q_{e}$ leads to complete suppression of the output coupling, while the case A, corresponding to conventional $2 \pi$-mode, restricts the increasement of $M^{2} R / Q$. As a result, $\alpha=0.8$ is adopted, with $M^{2} R / Q 59.69 \Omega$ (Case B) with a modest $Q_{e}$ of 2523.52 . In terms of electric field distribution, the wave-troughs amplitudes of $2 \pi$-like field component established in Case B (Fig. 6) can be tuned to near zero, which is the physical mechanism that provides a larger coupling coefficient $M$ to establish the more idealized strong-coupled $2 \pi$ mode (This can be derived by Eq. (2)).

To demonstrate the validity of the strong coupled $2 \pi$ mode established in $\mathrm{TM}_{31}$ operation mechanism, the 3D distributions formed by $\mathrm{z}$-directional electric field components $\left(E_{z}\right)$ are analyzed in Fig. 7(a) and 7(b), with length factors of $\alpha=0.8$ and $\alpha=1$ respectively. The 3D $E_{z}$ components at $\alpha=0.8$ are shown in Fig. 7(a), with a sinusoidal-like eight wave-crests and seven wave-troughs profile for the electric field along the beam tunnel. In short slots corresponding to the positions of wave-troughs, the uniform weak- $\pi$-phase fields can be observed, with the normalized amplitude defined as $\mathrm{A}_{1}$. In long slots corresponding to the wave-crests, the $2 \pi$-phase fields with normalized amplitude as $\mathrm{A}_{2}=6 \mathrm{~A}_{1}$ can play a major role in the speed modulation process. Such a sinusoidal-like profile of the electric field distribution is similar to the conventional $2 \pi$ mode case at $\alpha$ less than 0.24 (consistent with Fig. 6), but the waveform has moved closer to the $\pi$-mode and therefore provides a relatively high coupling coefficient. In this case, standing wave field in the region of both two coupling cavities needs to accommodate standing-wave field for $\lambda_{\mathrm{g}} / 2$ in $y$-axis to successfully establish the $\mathrm{TM}_{31}$ mode [20], where the waveguide wavelength $\lambda_{\mathrm{g}}$ can be obtained by

$$
\lambda_{g}=\frac{\lambda}{\sqrt{1-\left(\lambda / \lambda_{c}\right)^{2}}} .
$$

$\lambda$ and $\lambda_{\mathrm{c}}$ are the free-space wavelength and cut-off wavelength of the coupling cavities, respectively. Therefore, the coupling cavity height $c y$ should be slightly larger than $\lambda_{\mathrm{g}} / 2$ to ensure that the $\mathrm{TM}_{31}$ field resonance conditions can be satisfied. After a long simulation work, a $c y=0.69 \mathrm{~mm}$ was adopted, which is consistent with a value slightly larger than the calculated value
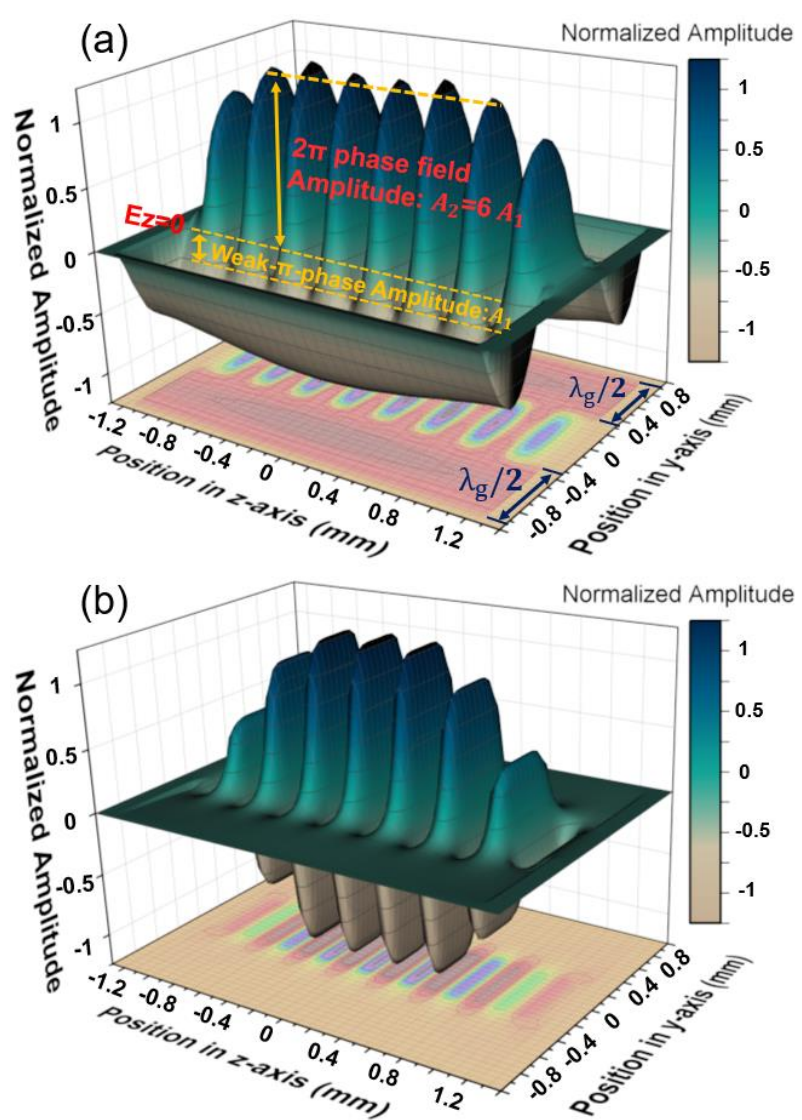

Fig. 7. Three-dimensional distribution diagrams of $E_{z}$ field for the overmoded EIO structure at length factors of (a) $\alpha=0.8$ and (b) $\alpha=1$, respectively.

of $0.65 \mathrm{~mm}$ for $\lambda_{\mathrm{g}} / 2$. As shown in Fig. 7(a), a standing wave field of $\lambda_{\mathrm{g}} / 2$ is excited in the coupling cavity, which represents successful establishment of the transversal over-dimensional $\mathrm{TM}_{31}$ mode, and provides the favorable coupling condition for the output structure.

On the other hand, for Fig. 7(b) at $\alpha=1$, there is no standing wave field exists in the coupling cavity in the case of uniformed interaction gaps. Since the output structure extracts energy from the coupling cavity, this case is not conducive to energy coupling, which is consistent with the analysis in Fig. 5. From above, the analysis of $3 \mathrm{D} E_{z}$ field components proves the validity of establishing a strongly coupled $\mathrm{TM}_{31}-2 \pi$ mode for a staggered bi-period structure.

Extensive particle-in-cell (PIC) simulations of the EIO have been carried out to demonstrate the performance of the circuit and its tolerance to injection conditions. The output power is extracted from a standard WR-2.8 waveguide through the coupling aperture which is above the middle of coupling cavity (Fig. 1). The stable output signal and particle energy distribution are obtained with an electron beam of $42.5 \mathrm{kV}$ and 0.6 A, as shown in Fig. 8(a) and 8(b). A stable output power of $540 \mathrm{~W}$ and an electronic efficiency of $2.1 \%$ is obtained at $351.92 \mathrm{GHz}$. We have also considered the effect of copper surface roughness on the output in our calculations, and by slightly increasing the injection current we can achieve an efficiency comparable to that in Fig. 8(a). Fig. 9 shows the circuit output tolerance-performance as a function of the beam voltage and energy spread of the electron beam. Promising 

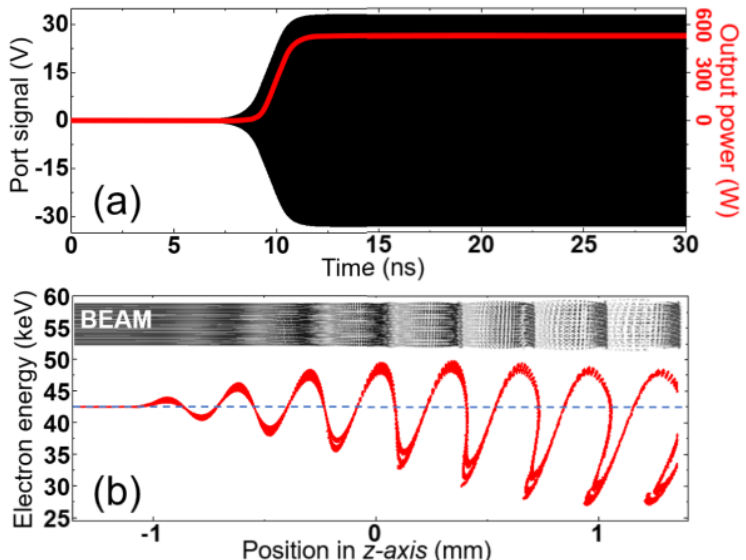

Fig. 8. (a) Time-dependent output signal and (b) electron energy distribution at saturation from CST-MWS simulations.

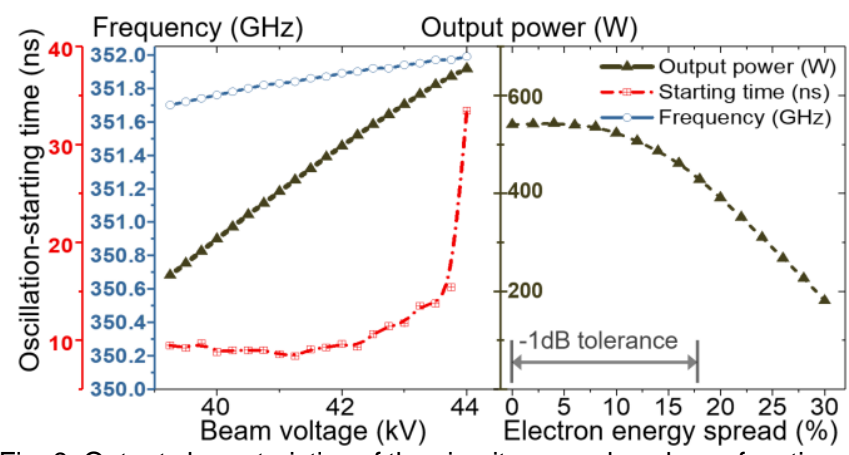

Fig. 9. Output characteristics of the circuit are analyzed as a function of beam voltage and energy spread, respectively.

results are obtained, in which the circuit operating stably in the beam voltage range of 39.2-44 kV with electronic efficiencies of $1.0 \%$ to $2.5 \%$. Likewise, the circuit demonstrates a moderate tolerance in terms of electron energy spread, with a $-1 \mathrm{~dB}$ energy spread tolerance of $\sim 18 \%$.

\section{CONCLUSIONS}

In summary, an overmoded circuit with bi-periodic structure is proposed to support stable, high-efficiency THz-EIO sources. The operation strong coupled $2 \pi$ mode is tuned by a staggered short/long slot mechanism that allows the presence of weakly $\pi$-phase field components in the short slot. According to extensive simulations and calculations, the proposed circuit demonstrates the advantages of both $\pi$ mode and $2 \pi$ mode in terms of coupling ability and output performance respectively. From these characteristics, a state-of-the-art $M^{2} R / Q$ value of $59.69 \Omega$ is achieved for a THz ladder-type extended interaction structure, with an electronic efficiency of $2.1 \%$ at $0.35 \mathrm{THz}$ in PIC simulations. The 3D analysis for the $E_{z}$ field verifies the validity of the staggered bi-periodic structure under $\mathrm{TM}_{31}$ operating mechanism that provides the over-sized structure.

The circuit has a slight additional processing difficulty compared to conventional EIO and shows a moderate tolerance for beam voltage and electron energy spread. Mode characteristics were analyzed, showing the advantage of stable operation and avoiding parasitic modes. The concept of mode tuning between $\pi$ and $2 \pi$ mode is attractive for satisfying stable operation and enhancing beam-wave interaction performance of THz-EIDs, especially for high-order $\left(\mathrm{TM}_{\mathrm{n} 1}\right)$ mode operation.

\section{REFERENCES}

[1] D. Berry, H. Deng, R. Dobbs, P. Horoyski, M. Hyttinen, A. Kingsmill, R. MacHattie, A. Roitman, E. Sokol, and B. Steer, "Practical aspects of eik technology," IEEE Transactions on Electron Devices, vol. 61, pp. 1830-1835, 06/01, 2014. doi: 10.1109/TED.2014.2302741.

[2] D. Chernin, A. Burke, I. Chernyavskiy, and J. Petillo, "12.3: Extended interaction klystrons for terahertz power amplifiers," In Proc. IEEE International Vacuum Electronics Conference, 2010. doi: 10.1109/IVELEC.2010.5503526.

[3] R. Dobbs, and B. Steer, "Extended interaction sources above $220 \mathrm{ghz}$," in 2011 International Conference on Infrared, Millimeter, and Terahertz Waves, 2011, pp. 1-1. doi: 10.1109/irmmw-THz.2011.6105059.

[4] J. H. Booske, R. J. Dobbs, C. D. Joye, C. L. Kory, G. R. Neil, G.-S. Park, J. Park, and R. J. Temkin, "Vacuum electronic high power terahertz sources," IEEE Transactions on Terahertz Science and Technology, vol. 1, no. 1, pp. 54-75, 2011. doi: 10.1109/TTHZ.2011.2151610.

[5] S. Dhillon, M. Vitiello, E. Linfield, A. Davies, M. C. Hoffmann, J. Booske, C. Paoloni, M. Gensch, P. Weightman, and G. Williams, "The 2017 terahertz science and technology roadmap," Journal of Physics D: Applied Physics, vol. 50, no. 4, pp. 043001, 2017. doi: 10.1088/1361-6463/50/4/043001.

[6] R. G. Carter, "Determination of the interaction impedance of helix slow-wave structures," in Abstracts. International Vacuum Electronics

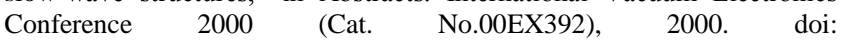
10.1109/ove:Ec. 2000.847509.

[7] G. X. Shu, H. Yin, L. Zhang, J. P. Zhao, G. Liu, A. D. R. Phelps, A. W. Cross, and W. He, "Demonstration of a planar w-band, kw-level extended interaction oscillator based on a pseudospark-sourced sheet electron beam," IEEE Electron Device Letters, vol. 39, no. 3, pp. 432-435, Mar, 2018. doi: 10.1109/Led.2018.2794469.

[8] Y. Yin, W. He, L. Zhang, H. Yin, C. W. Robertson, and A. W. Cross, "Simulation and experiments of a w-band extended interaction oscillator based on a pseudospark-sourced electron beam," IEEE Transactions on Electron Devices, vol. 63, no. 1, pp. 512-516, 2016. doi: 10.1109/ted.2015.2502950.

[9] C. Xu, L. Meng, C. F. Hu, Y. Yin, S. R. Zhu, Z. W. Chang, L. J. Bi, R. B. Peng, B. Wang, H. L. Li, and X. S. Yuan, "Analysis of dual-frequency radiation from a g-band extended interaction oscillator with double sheet beam," IEEE Transactions on Electron Devices, vol. 66, no. 7, pp. 3184-3189, Jul, 2019. doi: 10.1109/Ted.2019.2918191.

[10] K. Nguyen, E. Wright, V. Jabotinski, D. Pershing, L. Ludeking, P. Horoyski, A. Roitman, R. Dobbs, M. Hyttinen, D. Berry, D. Chernin, A. Burke, J. Petillo, J. Calame, B. Levush, and J. Pasour, "15.4: Design of terahertz extended interaction klystrons," in 2010 IEEE International Vacuum Electronics Conference (IVEC), 2010, pp. 327-328. doi: 10.1109/IVELEC.2010.5503416.

[11] Y. M. Shin, J. X. Wang, L. R. Barnett, and N. C. Luhmann, "Particle-in-cell simulation analysis of a multicavity w-band sheet beam klystron," IEEE Transactions on Electron Devices, vol. 58, no. 1, pp. 251-258, 2011. doi: 10.1109/ted.2010.2082544.

[12] C. Xu, L. Meng, Y. Yin, Z. Chang, L. Bi, R. Peng, B. Wang, and H. Li, "Demonstration of the electronic cutoff field in millimeter-wave extended interaction oscillators," IEEE Transactions on Electron Devices, pp. 1-7, 2021. doi: 10.1109/TED.2021.3066088.

[13] D. Wang, G. Wang, J. Wang, S. Li, P. Zeng, and Y. Teng, "A high-order mode extended interaction klystron at 0.34 thz," Physics of Plasmas, vol. 24 no. 2, 2017. doi: 10.1063/1.4975649.

[14] R. Li, C. Ruan, S. Li, and H. Zhang, "G-band rectangular beam extended interaction klystron based on bi-periodic structure," IEEE Transactions on Terahertz Science and Technology, vol. 9, no. 5, pp. 498-504, 2019. doi: 10.1109/tthz.2019.2927857.

[15] X. Ren, J. Wang, K. Dong, G. Liu, G. Shu, H. Fu, and E. A. Balfour, "Study of a high-efficiency 34-ghz sheet beam extended interaction oscillator with low filling factor," IEEE Transactions on Electron Devices, vol. 63, no. 10, pp. 4074-4080, 2016. doi: 10.1109/TED.2016.2595596.

[16] S. Li, C. Ruan, A. K. Fahad, P. Wang, Z. Zhang, and W. He, "Novel coupling cavities for improving the performance of g-band ladder-type multigap extended interaction klystrons," IEEE Transactions on Plasma Science, vol. 48, no. 5, pp. 1350-1356, 2020. doi: $10.1109 /$ tps.2020.2982957. 
[17] D. Zhao, W. Gu, X. Hou, G. Liu, Q. Xue, and Z. Zhang, "Demonstration of a high-power ka-band extended interaction klystron," IEEE Transactions on Electron Devices, vol. 67, no. 9, pp. 3788-3794, 2020. doi: 10.1109/TED.2020.3008881.

[18] J. Pasour, E. Wright, K. T. Nguyen, A. Balkcum, F. N. Wood, R. E. Myers, and B. Levush, "Demonstration of a multikilowatt, solenoidally focused sheet beam amplifier at $94 \mathrm{ghz}$," IEEE Transactions on Electron Devices, vol. 61, no. 6, pp. 1630-1636, 2014. doi: 10.1109/TED.2013.2295771.

[19] Z. Chang, L. Meng, Y. Yin, B. Wang, H. Li, L. Bi, R. Peng, and C. Xu, "Study of a dual-mode w-band extended interaction oscillator," IEEE Transactions on Electron Devices, vol. 65, no. 6, pp. 2620-2625, 2018. doi: 10.1109/TED.2018.2825105.

[20] L. Bi, L. Meng, J. Gong, R. Peng, Z. Chang, C. Xu, B. Wang, H. Li, P. Zhang, and Y. Yin, "Three-dimensional electromagnetic characteristic of overmoded coupling pattern for the cut-off extended interaction field in thz sheet beam resonant system," Journal of Physics D: Applied Physics, vol. 53, no. 13, pp. 135501, 2020. doi: 10.1088/1361-6463/ab6466.

[21] C. D. Joye, A. M. Cook, J. P. Calame, D. K. Abe, A. N. Vlasov, I. A. Chernyavskiy, K. T. Nguyen, E. L. Wright, D. E. Pershing, T. Kimura, M. Hyttinen, and B. Levush, "Demonstration of a high power, wideband 220-ghz traveling wave amplifier fabricated by uv-liga," IEEE Transactions on Electron Devices, vol. 61, no. 6, pp. 1672-1678, 2014. doi: 10.1109/TED.2014.2300014.

[22] S. Li, J. Wang, G. Wang, and D. Wang, "Theoretical studies on stability and feasibility of 0.34 thz eik," Physics of Plasmas, vol. 24, no. 5, pp. 053107, 2017/05/01, 2017. doi: 10.1063/1.4983621.

[23] D. Y. Wang, G. Q. Wang, J. G. Wang, S. Li, P. Zeng, and Y. Teng, "A high-order mode extended interaction klystron at 0.34 thz," Physics of Plasmas, vol. 24, no. 2, Feb, 2017. doi: Artn 023106

10.1063/1.4975649.

[24] G. Burt, L. Zhang, D. A. Constable, H. B. Yin, C. J. Lingwood, W. L. He, C. Paoloni, and A. W. Cross, "A millimeter-wave klystron upconverter with a higher order mode output cavity," IEEE Transactions on Electron Devices, vol. 64, no. 9, pp. 3857-3862, Sep, 2017. doi: 10.1109/Ted.2017.2724581.

[25] CST Microwave Studio, CST, Darmstadt, Germany, 2014. 